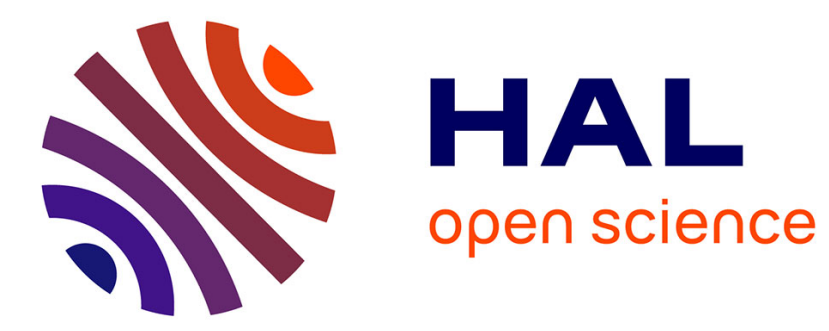

\title{
An Antenna Array for Photonic Beam Switching in Mm-Wave Wireless Communications.
}

Alvaro J Pascual, Fabien Ferrero, Laurent Brochier, Thomas Batté, Olivier de Sagazan, Guillermo Carpintero, Ronan Sauleau, D. Gonzalez-Ovejero

\section{- To cite this version:}

Alvaro J Pascual, Fabien Ferrero, Laurent Brochier, Thomas Batté, Olivier de Sagazan, et al.. An Antenna Array for Photonic Beam Switching in Mm-Wave Wireless Communications.. 15th European Conference on Antennas and Propagation (EUCAP 2021), Mar 2021, Dusseldorf, Germany. pp.1-4, 10.23919/EuCAP51087.2021.9410948 . hal-03137064

\section{HAL Id: hal-03137064 https://hal.science/hal-03137064}

Submitted on 27 Nov 2021

HAL is a multi-disciplinary open access archive for the deposit and dissemination of scientific research documents, whether they are published or not. The documents may come from teaching and research institutions in France or abroad, or from public or private research centers.
L'archive ouverte pluridisciplinaire HAL, est destinée au dépôt et à la diffusion de documents scientifiques de niveau recherche, publiés ou non, émanant des établissements d'enseignement et de recherche français ou étrangers, des laboratoires publics ou privés. 


\title{
An Antenna Array for Photonic Beam Switching in mm-Wave Wireless Communications
}

\author{
A. J. Pascual ${ }^{1}$, F. Ferrero ${ }^{2}$, L. Brochier ${ }^{2}$, T. Batté ${ }^{3}$, O. de $\operatorname{Sagazan}^{1}$, G. Carpintero ${ }^{4}$, R. Sauleau ${ }^{1}$, and D. González ${ }^{1}$ \\ ${ }^{1}$ Univ Rennes, CNRS, IETR (Institut d'Électronique et des Technologies du numéRique) - UMR 61614, F-35000, Rennes, \\ France, alvaro-jose.pascual@univ-rennes1.fr \\ ${ }^{2}$ Université Côte d'Azur, CNRS, LEAT (Laboratory of Electronics Antennas and Telecommunications) - UMR 7248, 06903 \\ Sophia Antipolis, France \\ ${ }^{3}$ Institut Foton, INSA (Institut National des Sciences Appliquées) - UMR 6082, Rennes, France \\ ${ }^{4}$ Universidad Carlos III de Madrid, Leganés, Spain
}

\begin{abstract}
We present a photonic transmitter at E-band for beam switching in $\mathrm{mm}$-wave wireless links. First, we discuss the trade-offs between beam switching and beam steering with arrays of photonic transmitters. Then, the designed transmitter is presented; it includes two $2 \times 2$ sub-arrays of stacked patches fed independently by two photodiodes. The transmitter is placed at the focal plane of a PTFE lens to increase the overall gain. Excitation of either sub-array allows 1D beam witching between $\pm 2.7^{\circ}$ for accurate beam alignment or reconfigurable links.
\end{abstract}

Index Terms-Antenna array, beam switching, beam steering, E-band, mm-waves, wireless communications, photomixing.

\section{INTRODUCTION}

The increasing demand for data traffic in the next generation of wireless systems will require the deployment of high-capacity cellular networks. The solutions foreseen to cope with this rapid growth of traffic include using larger bandwidths for multi-Gbps data throughput and network densification [1]. Indeed, wide bandwidths are already available at millimeter waves (mm-wave). In particular, the E-band (71-76 GHz and 81-86 GHz) constitutes one of the most promising candidates, since it offers a $2 \times 5 \mathrm{GHz}$ bandwidth within a relatively low atmospheric absorption window of approximately $0.5 \mathrm{~dB} / \mathrm{km}$ [2]. This provides about $30 \mathrm{~dB}$ extra margin in the link budget compared to the $60 \mathrm{GHz}$ band for $2 \mathrm{~km}$ wireless links [3].

One of the most promising technologies to generate the high frequency signals is the combination of photonic generation with Radio over fiber (RoF). In a nutshell, the radio signal is used to modulate two optical tones, whose frequency difference equals that of the $\mathrm{mm}$-wave carrier. The optical signal can be processed and transported to a highspeed opto-electronic (O/E) converter, such as a highbandwidth photodiode (PD). This photodiode is directly integrated with an antenna that radiates a modulated $\mathrm{mm}$ wave carrier. With a bandwidth spanning over $4 \mathrm{THz}$, photonic generation of mm-wave carriers can benefit from ultra-wide bandwidths and reliable and cost-effective commercial components, such as Er-Doped Fiber Amplifiers (EDFAs). On the downside, photonic generation yields less power than its electronic counterparts and high gain antennas $(>25 \mathrm{dBi})$ are required in $\mathrm{mm}$-wave wireless links [4].

The development of beam steering with high gain antennas will be a crucial aspect to encourage the use of photonic transmitters for e.g. reconfigurable outdoor/indoor links [4], to compensate twist and sways of supporting structures [5], or to simply ease alignment [6].

Although antennas fed by photonic sources have been reported extensively, just a few beam steering demonstrations with phased arrays may be found in the open literature [7]-[9]. In [7], beam steering was demonstrated using an array of broadband antennas integrated with a Silens. However, the antennas were electrically large and radiated to a high permittivity substrate $(\varepsilon r \sim 12)$ which caused an early onset of grating lobes. In [8] an array of $1 \times 4$ horn antennas were used but the low number of elements yielded a gain of $18 \mathrm{dBi}$ only. Similarly, in [9], the difficulty of illuminating uniformly several elements lead to a $1 \times 3$ array with reduced gain and beam scan. The experimental challenge of integrating many array elements efficiently illuminated has hindered improvements towards the realization of practical arrays with high gain and beam steering simultaneously.

In this manuscript, we first propose and discuss the advantages of beam switching as an alternative to phased array of photonic transmitters. Then, we explain the designed structure and show simulation results where high gain and a steerable beam (in discrete directions) are simultaneously accomplished.

\section{PHOTONIC BEAM SWITCHING}

Fig. 1a shows a typical block diagram of a photonic transmitter for a phased array [8], whereas Fig. 1b shows the equivalent for the beam switching alternative adopted in this paper. In phased arrays, the optical tones modulated according to a data string are carried by an optical fiber (yellow line). The optical input is then divided among the $N$ elements of the array. For a phased array it is compulsory to control the amplitude and phase of the signal driving each array element. This is accomplished in the optical domain with a variable optical attenuator (VOA) or amplifier, and an 


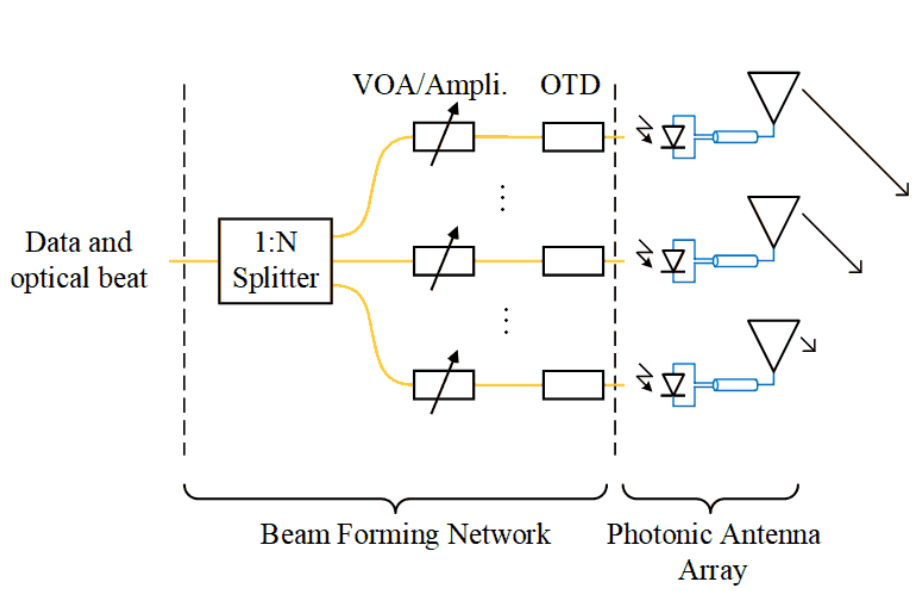

(a)

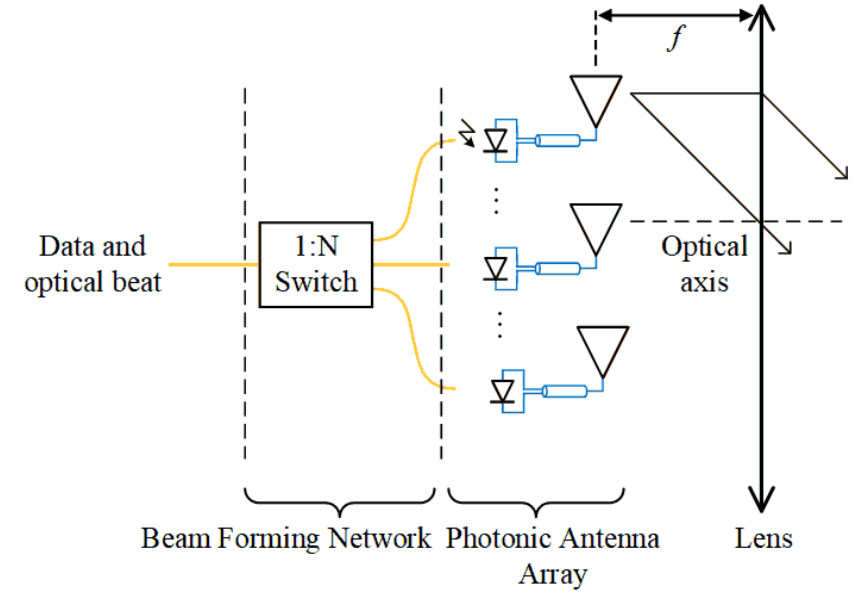

(b)

Fig. 1 Block diagram and ray tracing of an array of photonic transmitters in (a) a phased array and (b) for beam switching.

optical time delay (OTD). After the VOA-OTD chain, the light illuminates the photodiodes, in turn coupled to the antennas (blue line) that will radiate the modulated mm-wave carrier in the desired direction.

On the other hand, for photonic beam switching, $N$ antennas are located at the back focal plane of a lens with focal distance $f$. For an antenna placed at a distance $s$ out of the optical axis, the beam tilt is given from geometrical optics by:

$$
\tan \theta= \pm s / f
$$

where $\theta$ is the angle measured respect to broadside. In this case, only one antenna is active at a time, and the beam can be switched among $N$ different directions governed by (1). Compared with a phased array, this technique presents several trade-offs, that make it an extremely interesting route to explore.

First, beam switching can achieve high gain (due to the lens) using a reduced number of elements. For instance, a square array of half-wavelength dipoles over a ground plane, uniformly excited and spaced $\lambda_{0} / 2$, presents a maximum gain of $19.1 \mathrm{dBi}$ for 25 elements and $25.2 \mathrm{dBi}$ for 100 elements. On the downside, beam switching allows discrete scanning directions only, and the pattern degradation when $s$ is comparable to $f$ or lens diameter limits the scan range.

Besides, the grating lobe condition of an array disappears, so the element spacing is taken as a design variable and it can be in principle larger than $\lambda_{0} / 2$. This is a crucial characteristic for photonic transmitters where each of the PD is illuminated by an optical fiber precisely aligned. A larger separation between elements also eases the integration of the PD with RF amplifiers or matching circuits.

Finally, the beam forming network is largely simplified respect to that of a phased array, and beam switching can be performed using low insertion loss and low power consuming optical switches.

\section{MM-WAVE PhOTONIC TRANSMitTER}

Fig. 2 shows the proposed photonic transmitter concept. It is fabricated using Printed Circuit Board (PCB) technology on a $127 \mu \mathrm{m}$-thick, high-frequency Duroid ${ }^{\circledR} 5880$ substrate $\left(\varepsilon_{\mathrm{r}}=2.24, \tan \delta=0.004\right.$ at $\left.60 \mathrm{GHz}\right)[10]$, depicted in brown. It consists of two independent sub-arrays composed of four

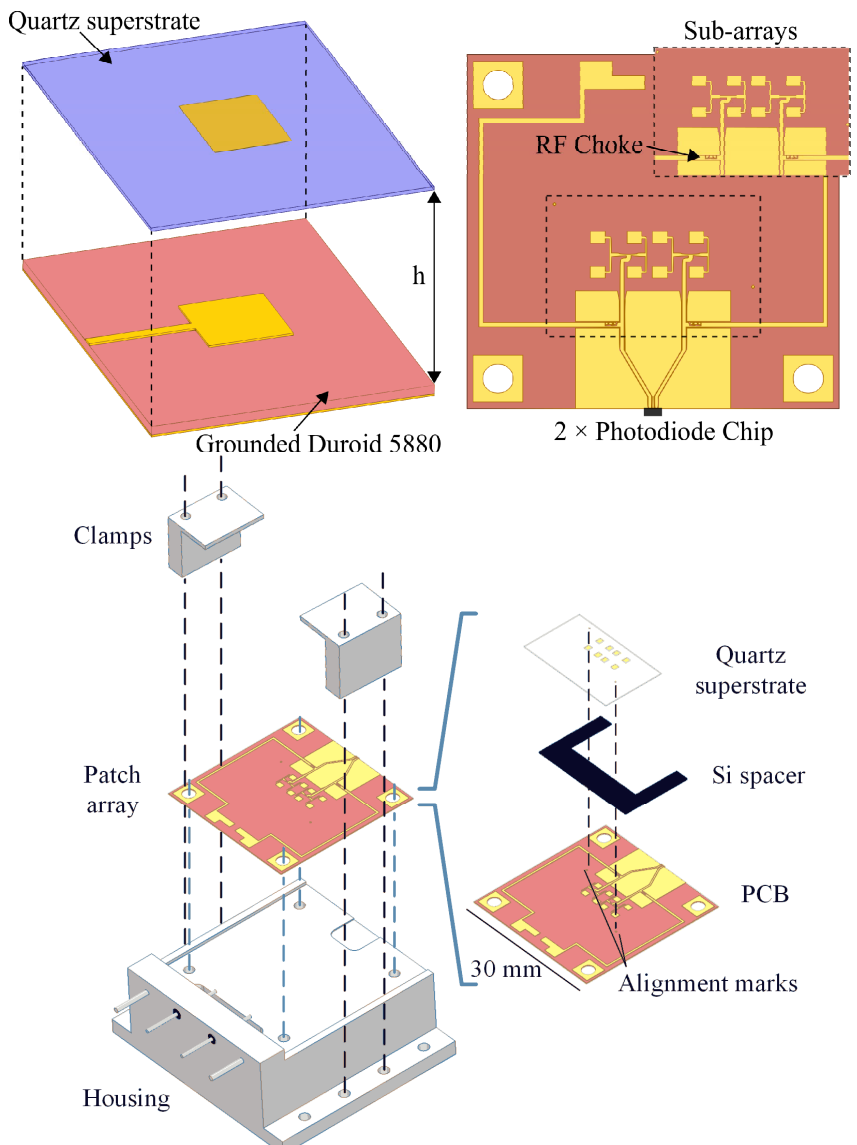

Fig. 2 Top left: the primary sub-array radiator consist of a stack of two patches, one printed directly on the PCB and fed by a microstrip line, and other on a quartz superstrate. Top right: designed PCB. Bottom: structure of the antenna with the top and bottom patches separated by a silicon spacer. 
stacked patches, each sub-array is integrated with a photodiode (PD) as in [11].

The design of the transmitter has been carried out with High Frequency Structure Simulator (ANSYS HFSS, release 19.0.0) and starts with the optimization of the primary radiator, for which we have selected stacked patches [12]. This approach usually consists in using a driven patch and a coupled parasitic patch with similar dimensions and placed at a certain height $h$ from the driven patch, as in the top left of Fig. 2. The overall structure presents two close resonances, thereby enhancing the impedance bandwidth of the single microstrip patch. In this case, the bottom patch is directly printed on the PCB whereas the top patch is printed on the bottom side of a $100 \mu \mathrm{m}$-thick quartz superstrate. Quartz exhibits low loss $\left(\tan \delta<0.005\right.$ in W-band), relatively low $\varepsilon_{\mathrm{r}}$ $(\sim 3.80)$ and is optically transparent. Therefore, alignment marks on both the PCB and the quartz enable an accurate positioning of the patches (Fig. 2 bottom). The distance $h$ between the top and the bottom patches is accurately controlled by a silicon spacer, which can be polished until the desired height. For the proposed configuration, we have achieved a $23 \%$ relative bandwidth with reflection coefficient below $-10 \mathrm{~dB}$ for an optimum $h$ of $300 \mu \mathrm{m}$. Besides, the extended coupled cavity in the vertical direction does not increase the area of the element, and preserves the symmetry of the structure. Hence, it maintains the radiation pattern and the phase center, which are desirable characteristics for array configurations used as primary feed in a quasi-optical system.

Once the stacked patch has been designed, it is replicated to form the two $2 \times 2$ sub-arrays observed in Fig. 2 top right. A feed network divides the incoming signal from the $50-\Omega$ microstrip line into the four $80-\Omega$ microstrip lines that feed each of the patches.

Regarding the feed network connecting the photodiodes with the antenna, a tapered transition is first used to adapt the coplanar waveguide (CPW) output from the photodiodes grown on InP to the Grounded-CPW on Duroid®. Next, both signal lines separate to reach the distance required to feed the $2 \times 2$ sub-arrays. Perpendicular to the signal lines, one can also observe in the top right of Fig. 2 the RF chokes made of

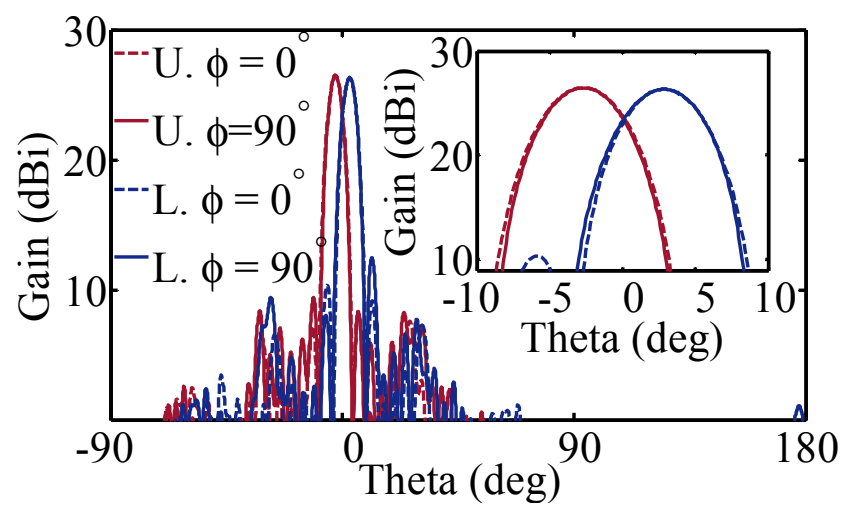

Fig. 3 Values of simulated gain for the upper (U.) and lower (L.) subarrays. The continuous line corresponds to the E-plane of the patches and the dashed line to the H-plane. split-ring resonators [13] and the DC interconnections used to bias the photodiodes. These chokes feature an isolation higher than $20 \mathrm{~dB}$ on the whole E-band. Last, transitions from $\mathrm{G}-\mathrm{CPW}$ to $50-\Omega$ microstrip lines are used to excite the corporate feed network in each $2 \times 2$ sub-array.

\section{RESUlTS}

To demonstrate beam switching, the transmitter is placed at the focal plane of a PTFE lens (Thorlabs LAT075) with a back focal length of $53 \mathrm{~mm}$ and diameter of $5 \mathrm{~cm}$. The two sub-arrays lie symmetrically above and below, out of the optical axis $2.5 \mathrm{~mm}$. Therefore, from (1), excitation of one of either photodiodes produces a beam at $\theta \approx \pm 2.7^{\circ}$. Fig. 3 shows the simulation results of the transmitter plus lens for $80 \mathrm{GHz}$ using ANSYS HFSS' Finite Element-Boundary Integral (FE-BI) boundary conditions. Both beams cross at $-3 \mathrm{~dB}$, and the pointing direction is stable across the E-band. Note the antenna can be mirrored and scaled in 1-D to obtain $2 \times \mathrm{N}$ beam directions.

\section{CONCLUSIONS}

We have discussed the benefits of beam switching over beam steering with arrays of photonic transmitters in mmwave wireless links. A practical realization of photonic beam switching has been also presented. The proposed solution consists of two $2 \times 2$ sub-arrays of stacked patches fed independently by two photodiodes. The sub-arrays are placed at the focal plane of a PTFE lens that enhances the overall gain, while excitation of either sub-array allows 1D beam witching between $\pm 2.7^{\circ}$. This scanning range enables accurate beam alignment or the deployment of reconfigurable links. A prototype has been assembled, and measurements of radiation patterns and return loss are ongoing. These measurements will be presented at the conference, along with the assessment of the transmitter performance in a reconfigurable wireless communications link.

\section{ACKNOWLEDGMENT}

The authors would like to thank the European Union financement through the European Regional Development Fund (ERDF), and the French region of Brittany, Ministry of Higher Education and Research, Rennes Métropole and Conseil Départemental 35, through the CPER Project SOPHIE / STIC \& Ondes. They also thank Labex CominLabs, funding by ANR, under project WASSAP (Wireless At Sub-millimeter waves: Signals, Antennas and Photonics).

\section{REFERENCES}

[1] J. G. Andrews et al., "What will 5G be?," in IEEE J. Sele. Areas Commun., vol. 32, no. 6, pp. 1065-1082, June 2014.

[2] 'Millimeter wave propagation: spectrum management' Federal Communications Commission, Office of Engineering and Technology, Bull. 70 (1997). 
[3] A. Stöhr et al., "Robust 71-76 GHz radio-over-fiber wireless link with high-dynamic range photonic assisted transmitter and laser phase-noise insensitive SBD receiver," OFC 2014, San Francisco, CA, 2014, pp. 1-3.

[4] H. Song, S. Priebe, and T. Kürner Handbook, "THz wireless communications," in Handbook of Terahertz Technologies, 1st ed. H. Song and T. Nagatsuma, Eds. Pan Stanford Publishing, 2016.

[5] R. Kalimulin, A. Artemenko, R. Maslennikov, J. Putkonen, and J. Salmelin, "Impact of mounting structures twists and sways on pointto-point millimeter-wave backhaul links," IEEE Int. Conf. Communication Workshop (ICCW), London, 2015, pp. 19-24.

[6] A. Hirata et al., "Transmission trial of television broadcast materials using 120-GHz-band wireless link," NTT, Atsugi-shi, Japan, Tech. Review, vol. 7, no. 3, Mar. 2009.

[7] S. Preu et al., "Fiber-Coupled 2-D n-i-pn-i-p superlattice photomixer Array," IEEE Trans. Antennas Propag., vol. 65, no. 7, pp. 3474-3480, July 2017.

[8] T. McKenna, J. Nanzer, and T. Clark Jr., "Photonic beamsteering of millimeter-wave array with $10-\mathrm{Gb} / \mathrm{s}$ data transmission," IEEE Photon. Technol. Lett., vol 26, no. 14, pp. 1407-1410, July 2014.

[9] N. Shimizu and T. Nagatsuma, "Photodiode-integrated microstrip antenna array for subterahertz radiation," IEEE Photon. Technol. Lett., vol. 18, no. 6, pp. 743-745, March 2006.

[10] O. Lafond, and M. Himdi, "Printed Millimeter Antennas- Multilayer Technologies," in Advanced Millimeter-Wave Technologies, 1st ed. D. Liu, B. Gaucher, U. Pfeiffer, and J. Grzyb, Eds. John Wiley \& Sons, Ltd., 2009.

[11] A. Pascual-Gracia et al., "A photonically excited leaky-wave antenna array at E-band for 1-D beam steering," Appl. Sci., 10, 3474, May 2020.

[12] D. M. Pozar, and D. H. Schaubert, "Techniques for improving element bandwidth," in Microstrip antennas: The analysis and design of microstrip antennas and arrays, IEEE, 1995, pp.155-201.

[13] M. Ali, L. E. G. Muñoz, and G. Carpintero, "E-band photonic transmitter with tapered slot antenna for RoF applications," Int. Topical Meeting Microw. Photon. (MWP), Beijing, 2017, pp. 1-4. 